\title{
Uncertainty in the environmental modelling process - A framework and guidance
}

\author{
Jens Christian Refsgaard ${ }^{a, *}$, Jeroen P. van der Sluijs ${ }^{b}$, Anker Lajer Højberg ${ }^{a}$, \\ Peter A. Vanrolleghem ${ }^{\mathrm{c}, \mathrm{d}}$ \\ ${ }^{a}$ Geological Survey of Denmark and Greenland (GEUS), Copenhagen, Denmark \\ ${ }^{\mathrm{b}}$ Copernicus Institute for Sustainable Development and Innovation, Department of Science Technology and Society, \\ Utrecht University, Utrecht, The Netherlands \\ ${ }^{\mathrm{c}}$ BIOMATH, Ghent University, Gent, Belgium \\ ${ }^{\mathrm{d}}$ Water Quality Modelling modelEAU, University Laval, Quebec, Canada
}

Received 20 December 2005; received in revised form 5 February 2007; accepted 7 February 2007

Available online 27 April 2007

\begin{abstract}
A terminology and typology of uncertainty is presented together with a framework for the modelling process, its interaction with the broader water management process and the role of uncertainty at different stages in the modelling processes. Brief reviews have been made of 14 different (partly complementary) methods commonly used in uncertainty assessment and characterisation: data uncertainty engine (DUE), error propagation equations, expert elicitation, extended peer review, inverse modelling (parameter estimation), inverse modelling (predictive uncertainty), Monte Carlo analysis, multiple model simulation, NUSAP, quality assurance, scenario analysis, sensitivity analysis, stakeholder involvement and uncertainty matrix. The applicability of these methods has been mapped according to purpose of application, stage of the modelling process and source and type of uncertainty addressed. It is concluded that uncertainty assessment is not just something to be added after the completion of the modelling work. Instead uncertainty should be seen as a red thread throughout the modelling study starting from the very beginning, where the identification and characterisation of all uncertainty sources should be performed jointly by the modeller, the water manager and the stakeholders. (c) 2007 Elsevier Ltd. All rights reserved.
\end{abstract}

Keywords: Integrated water resources management; Water framework directive; Catchment modelling; Uncertainty

\section{Introduction}

New guidelines on water resources management emphasise the importance of integrated approaches, cross-sectoral planning and of public participation (GWP, 2000; EC, 2003; Jønch-Clausen, 2004). The commonly accepted approach integrated water resources management (IWRM) is defined as "a process, which promotes the co-ordinated development and management of water, land and related resources, in order to maximise the resultant economic and social welfare in an equitable manner without compromising the sustainability of

\footnotetext{
* Corresponding author. Tel.: +45 38142 776; fax: +45 38142050 .

E-mail address: jcr@geus.dk (J.C. Refsgaard).
}

vital ecosystems" (GWP, 2000). IWRM deals with complex problems involving technological, environmental, economical and societal aspects. In addition a wide range of uncertainties ranging from ambiguity in defining problems and goals to uncertainty in data and models have to be taken into account in the management process.

The fundamental importance of uncertainty in water management can be illustrated by EU's Water Framework Directive (WFD). The WFD is an outcome of EU environmental policy, where one of the basic principles is "to contribute to the pursuit of the objectives of preserving, protecting and improving the quality of the environment in prudent and rational use of natural resources, and to be based on the precautionary principle" (EC, 2000). As the precautionary principle aims to protect humans and the environment against uncertain risks by 
means of pre-damage control (anticipatory measures) it can not be implemented without incorporating uncertainty assessments into the decision making process.

Uncertainty assessment of model simulations is therefore important, when models are used to support water management decisions (Beven and Binley, 1992; Beven, 2002; Pahl-Wostl, 2002; Jakeman and Letcher, 2003; Refsgaard and Henriksen, 2004; Pahl-Wostl, 2007; Vandenberghe et al., 2007). Model uncertainty is in practice often done as an 'end of pipe' analysis that is carried out after model set-up, calibration and validation have been completed. For integration of model results into the broader water management process and to increase effectiveness of knowledge production and use, Refsgaard et al. (2005a) emphasise the importance of making uncertainty analyses an ongoing theme from the beginning with problem definition and identification of modelling objectives and then throughout the modelling process.

The objective of this paper is to analyse the need and role for uncertainty analyses at the various stages of the modelling process and to briefly review methodologies and tools suitable for these various types of uncertainty assessments. The paper focuses on uncertainty in the modelling process. As such it touches upon aspects of uncertainty related to the broader policy and public participation processes, but it does not intend to fully cover these broader aspects.

\section{Modelling as part of the planning and management process}

A modelling study will involve several phases and several actors. A typical modelling study will involve the following four different types of actors:

- The water manager, i.e. the person or organisation responsible for the management or protection of the water resources, and thus of the modelling study and the outcome (the problem owner).

- The modeller, i.e. a person or an organisation that develops the model and works it, conducting the modelling study. If the modeller and the water manager belong to different organisations, their roles will typically be denoted consultant and client, respectively.

- The reviewer, i.e. a person that is conducting some kind of external review of a modelling study. The review may be more or less comprehensive depending on the requirements of the particular case. The reviewer is typically appointed by the water manager to support her/him to match the modelling capability of the modeller.

- The stakeholders/public, i.e. an interested party with a stake in the water management issue, either in exploiting or protecting the resource. Stakeholders include the following categories: (1) competent water resource authority (typically the water manager, cf. above); (2) interest groups; and (3) general public.

The modelling process may, according to the HarmoniQuA project (Refsgaard et al., 2005a; Scholten et al., 2007, http:// www.harmoniqua.org), be decomposed into five major steps (Fig. 1), which again are decomposed into 48 tasks (not detailed here). The contents of the five steps are:

- STEP1 (model study plan). This step aims to agree on a Model Study Plan comprising answers to the questions: Why is modelling required for this particular model study? What is the overall modelling approach and which work should be carried out? Who will do the modelling work? Who should do the technical reviews? Which stakeholders/public should be involved and to what degree? What are the resources available for the project? The water manager needs to describe the problem and its context as well as the available data. A very important (but often overlooked) task is then to analyse and determine what are the various requirements of the modelling study in terms of the expected accuracy of modelling results. The acceptable level of accuracy will vary from case to case and must be seen in a socio-economic context. It should, therefore, be defined through a dialogue between the modeller, water manager and stakeholders/public. In this respect an a priori analysis of the key sources of uncertainty is crucial in order to focus the study on the elements that produce most information of relevance to the problem at hand.

- STEP 2 (data and conceptualisation). In this step the modeller should gather all the relevant knowledge about the study basin and develop an overview of the processes and their interactions in order to conceptualise how the system should be modelled in sufficient detail to meet the requirements specified in the model study plan. Consideration must be given to the spatial and temporal detail required of a model, to the system dynamics, to the boundary conditions and to how the model parameters can be determined from available data. The need to model certain processes in alternative ways or to differing levels of detail in order to enable assessments of model structure uncertainty should be evaluated. The availability of existing computer codes that can address the model requirements should also be evaluated.

- STEP 3 (model set-up). Model set-up implies transforming the conceptual model into a site-specific model that can be run in the selected model code. A major task in model setup is the processing of data in order to prepare the input files necessary for executing the model. Usually, the model is run within a graphical user interface (GUI) where many tasks have been automated.

- STEP 4 (calibration and validation). This step is concerned with the process of analysing the model that was constructed during the previous step, first by calibrating the model, and then by validating its performance against independent field data. Finally, the reliability of model simulations for the intended type of application is assessed through uncertainty analyses. The results are described so that the scope of model use and its associated limitations are documented and made explicit.

- STEP 5 (simulation and evaluation). In this step the modeller uses the calibrated and validated model to make 


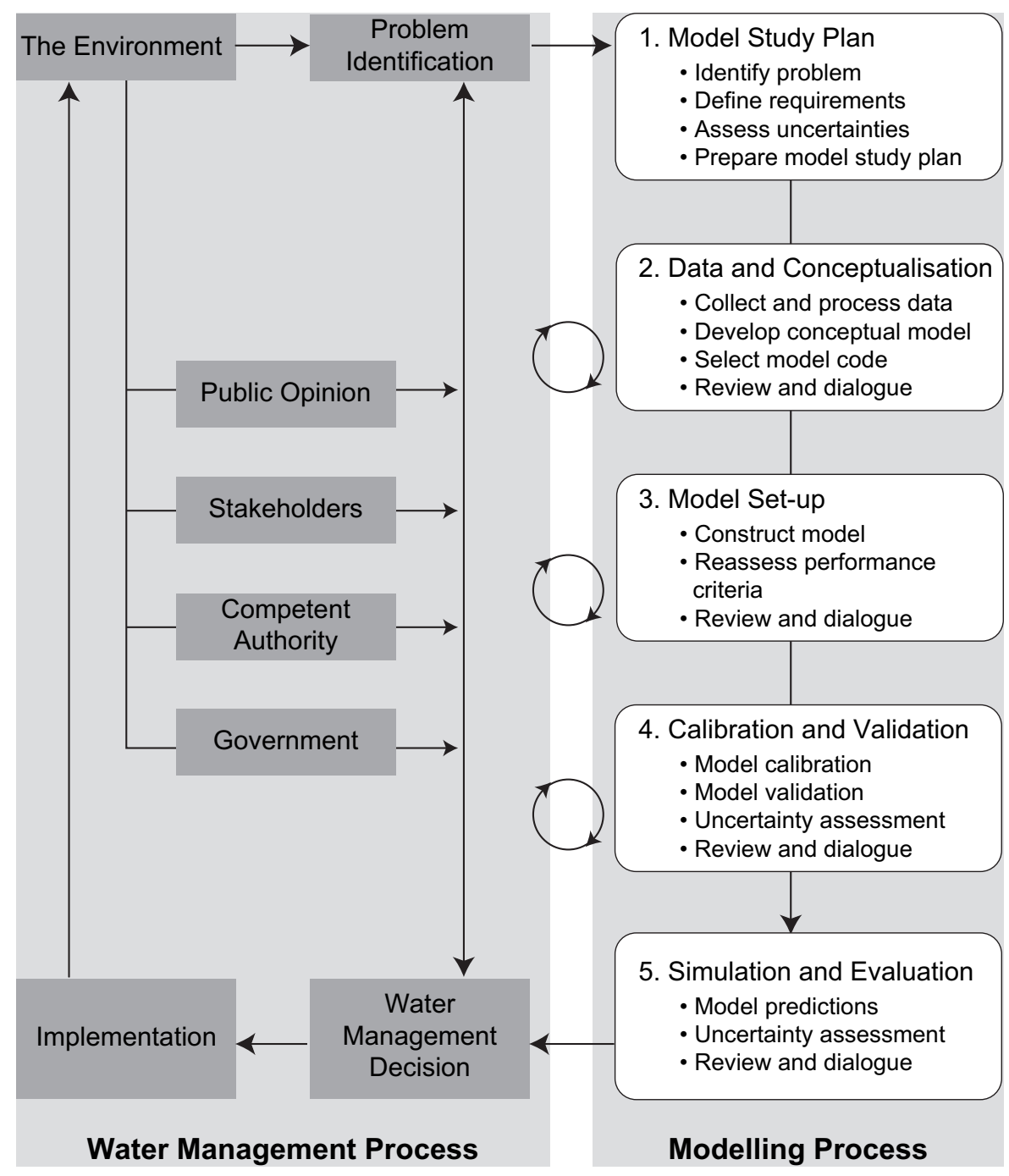

Fig. 1. The interactions between the five steps of the modelling process and the water management process (inspired from Refsgaard et al., 2005a and Pascual et al., 2003).

simulations to meet the objectives and requirements of the model study. Depending on the objectives of the study, these simulations may result in specific results that can be used in subsequent decision making (e.g. for planning or design purposes) or to improve understanding (e.g. of the hydrological/ecological regime of the study area). It is important to carry out suitable uncertainty assessments of the model predictions in order to arrive at a robust decision. As with the other steps, the quality of the results needs to be assessed through internal and external reviews that also provide platforms for dialogues between water manager, modeller, reviewer and, often, stakeholders/public.

Fig. 1 shows the key actors in the water management process and the above five steps in the modelling process. The interactions between the modelling process and the water management process are very clear at the beginning of the modelling process (Step 1), where the modeller receives the specifications of objectives and requirements for the modelling study from the water management process, and towards the end of the modelling study (Step 5), where the modelling results are provided as input to the water management process. These two interactions are usually participatory in the sense that not only the water manager, but also key stakeholders, are involved in the dialogue with the modeller. In this respect a participatory-based assessment of the most important sources of uncertainty for the decision process is important in Step 1 as a basis for prioritising the elements of the modelling study. During the main modelling process itself (Steps 2, 3,4) the link between the water management process and the modelling process consists of dialogue, reviews and discussions of preliminary results. The amount and type of interaction depend on the level of public participation that may vary from case to case, from providing information over consultation to active involvement (Henriksen et al., submitted for publication).

The typical cyclic and iterative character of the water management process, such as the WFD process, is illustrated in Fig. 2, where the interaction with the modelling process is illustrated by the large circle (water management) and the four smaller 
supporting circles (modelling). The WFD planning process, as most other planning processes, contains four main elements:

- Identification including assessment of present status, analysis of impacts and pressures and establishment of environmental objectives. Here modelling may be useful for example for supporting assessments of what are the reference conditions and what are the impacts of the various pressures.

- Designing including the set-up and analysis of a programme of measures designed to be able to reach the environmental objectives in a cost effective way. Here modelling will typically be used for supporting assessments of the effects and costs of various measures under consideration.

- Implementing the measures. Here real-time forecasting in some cases may support the operational decisions to be made.

- Evaluation of the effects of the measures on the environment. Here modelling may support the monitoring tasks in order to extract maximum information from the monitoring data, e.g. by indicating errors and inadequacies in the data and by filtering out the effects of climate variability.

\section{Uncertainty terminology and classification}

\subsection{Definitions and taxonomy}

Uncertainty and associated terms such as error, risk and ignorance are defined and interpreted differently by different authors, see Walker et al. (2003) for a review. The different definitions reflect the underlying scientific philosophical way of thinking and therefore typically vary among different scientific disciplines. In addition they vary depending on their

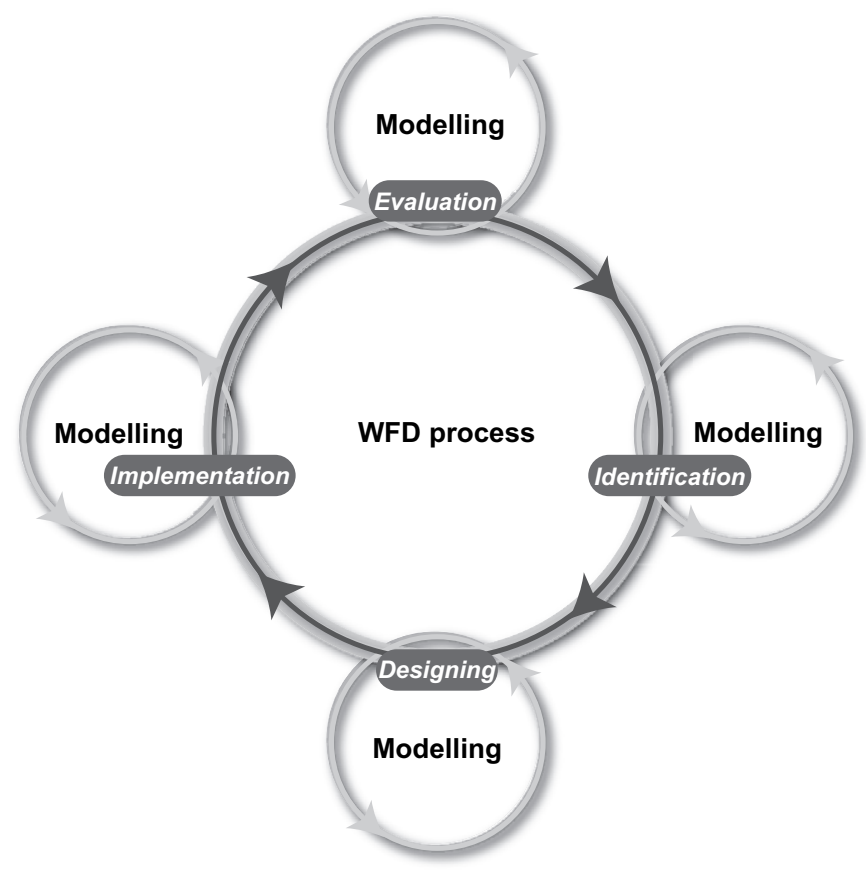

Fig. 2. The role of modelling in the water management process within the context of the EU Water Framework Directive (WFD). purpose. Some are rather generic, such as Funtowicz and Ravetz (1990), while others apply more specifically to model based water management, such as Beck (1987).

In this paper we will use the terminology of Klauer and Brown (2004) that has emerged after discussions between social scientists and natural scientists specifically aiming at applications in model based water management. By doing so we adopt a subjective interpretation of uncertainty in which the degree of confidence that a decision maker has about possible outcomes and/or probabilities of these outcomes is the central focus. Thus according to our definition a person is uncertain if s/he lacks confidence about the specific outcomes of an event. Reasons for this lack of confidence might include a judgement of the information as incomplete, blurred, inaccurate, unreliable, inconclusive, or potentially false. Similarly, a person is certain if $\mathrm{s} / \mathrm{he}$ is confident about the outcome of an event. It is possible that a person feels certain but has misjudged the information (i.e. his/her judgement is wrong).

There are many different decision situations, with different possibilities for characterising uncertainty. A first distinction is between ignorance as a lack of awareness that knowledge is wrong or imperfect, and uncertainty as a known degree of unreliability of knowledge, which translates into a state of confidence. In this respect Brown (2004) has defined a taxonomy of imperfect knowledge as illustrated in Fig. 3.

It is useful to distinguish between bounded uncertainty, where all possible outcomes are deemed 'known' and unbounded uncertainty, where some or all possible outcomes are deemed unknown. Since quantitative probabilities require 'all possible outcomes' of an uncertain event and each of their individual probabilities to be known, they can only be defined for 'bounded uncertainties'. If probabilities cannot be quantified in any undisputed way, we often can still qualify the available body of evidence for the possibility of various outcomes in terms of plausibility or convincingness of the evidence (e.g. Weiss, 2003). If outcomes but no probabilities are known we have to rely on 'scenario analysis'.

The bounded uncertainty where all probabilities are assumed known (the lower left case in Fig. 3) is often denoted 'statistical uncertainty' (e.g. Walker et al., 2003). This is the case that is traditionally addressed in model-based uncertainty assessments. It is important to note that this case only constitutes one of many of the decision situations outlined in Fig. 3, and, in many situations, the main uncertainty in a decision situation cannot be characterised quantitatively.

\subsection{Sources of uncertainty}

Walker et al. (2003) describe uncertainty as manifesting itself at different locations in the model-based water management process. These locations, or sources, may be characterised as follows:

- Context and framing, i.e. at the boundaries of the system to be modelled. The model context is typically determined at the initial stage of the study where the problem is identified and the focus of the model study selected as a confined part of the overall problem. This includes, for example, the 


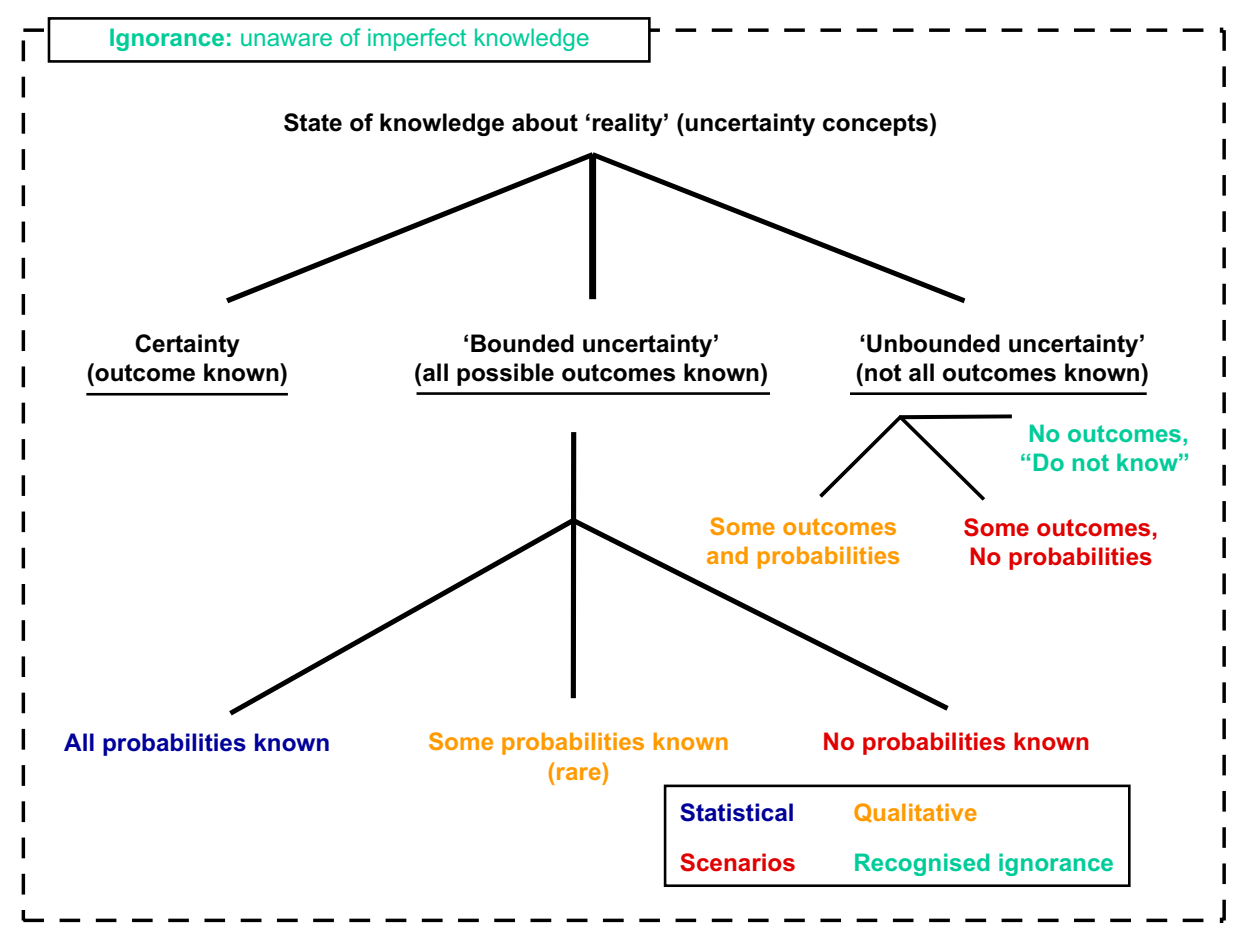

Fig. 3. Taxonomy of imperfect knowledge resulting in different uncertainty situations (Brown, 2004).

external economic, environmental, political, social and technological circumstances that form the context of the problem.

- Input uncertainty in terms of external driving forces (within or outside the control of the water manager) and system data that drive the model such as land use maps, pollution sources and climate data.

- Model structure uncertainty is the conceptual uncertainty due to incomplete understanding and simplified descriptions of modelled processes as compared to reality.

- Parameter uncertainty, i.e. the uncertainties related to parameter values.

- Model technical uncertainty is the uncertainty arising from computer implementation of the model, e.g. due to numerical approximations, resolution in space and time, and bugs in the software.

The total uncertainty on the model simulations, model output uncertainty, can be assessed by uncertainty propagation taken all the above sources into account.

\subsection{Nature of uncertainty}

Walker et al. (2003) explain that the nature of uncertainty can be categorised into:

- Epistemic uncertainty, i.e. the uncertainty due to imperfect knowledge.

- Stochastic uncertainty or ontological uncertainty, i.e. uncertainty due to inherent variability, e.g. climate variability.
Epistemic uncertainty is reducible by more studies, e.g. comprising research and data collection. Stochastic uncertainty is non-reducible.

Often the uncertainty on a certain event includes both epistemic and stochastic uncertainty. An example is the uncertainty of the 100 year flood at a given site. This flood event can be estimated: e.g. by use of standard flood frequency analysis on the basis of existing flow data. The (epistemic) uncertainty may be reduced by improving the data analysis, by making additional monitoring (longer time series) or by deepening our understanding of how the modelled system works. However, no matter how perfect both the data collection and the mechanistic understanding of the system are, and, no matter for how long historical data time series exist, there will always be some (stochastic) uncertainty inherent to the natural system, related to the stochastic and chaotic nature of several natural phenomena, such as weather. Perfect knowledge on these phenomena cannot give us a deterministic prediction, but would have the form of a perfect characterisation of the natural variability.

\subsection{The uncertainty matrix}

The uncertainty matrix in Table 1 can be used as a tool to get an overview of the various sources of uncertainty in a modelling study. The matrix is modified after Walker et al. (2003) in such a way that it matches Fig. 3 and so that the taxonomy now gives 'uncertainty type' in descriptions that indicate in what terms uncertainty can best be described. The vertical axis identifies the location or source of uncertainty while the horizontal axis covers the level and nature of uncertainty. 
Table 1

The uncertainty matrix (modified after Walker et al., 2003)

\begin{tabular}{|c|c|c|c|c|c|c|c|}
\hline \multirow{2}{*}{\multicolumn{2}{|c|}{ Source of uncertainty }} & \multicolumn{4}{|c|}{ Taxonomy (types of uncertainty) } & \multicolumn{2}{|l|}{ Nature } \\
\hline & & $\begin{array}{l}\text { Statistical } \\
\text { uncertainty }\end{array}$ & $\begin{array}{l}\text { Scenario } \\
\text { uncertainty }\end{array}$ & $\begin{array}{l}\text { Qualitative } \\
\text { uncertainty }\end{array}$ & $\begin{array}{l}\text { Recognised } \\
\text { ignorance }\end{array}$ & $\begin{array}{l}\text { Epistemic } \\
\text { uncertainty }\end{array}$ & $\begin{array}{l}\text { Stochastic } \\
\text { uncertainty }\end{array}$ \\
\hline Context & $\begin{array}{l}\text { Natural, technological, } \\
\text { economic, social, political }\end{array}$ & & & & & & \\
\hline \multirow[t]{2}{*}{ Inputs } & System data & & & & & & \\
\hline & Driving forces & & & & & & \\
\hline \multirow[t]{3}{*}{ Model } & Model structure & & & & & & \\
\hline & Technical & & & & & & \\
\hline & Parameters & & & & & & \\
\hline \multicolumn{2}{|c|}{ Model outputs } & & & & & & \\
\hline
\end{tabular}

It is noticed that the matrix is in reality three-dimensional (source, type, nature). Thus, the categories type and nature are not mutually exclusive, and it may be argued that the matrix should be modified in such a way that the two uncertainties within nature (epistemic and variability) should become subcells within the type categories. This is not done for graphical reasons.

\section{Methodologies for uncertainty assessment}

Many methodologies and tools suitable for supporting uncertainty assessment have been developed and reported in the scientific literature. We have selected 14 methods to represent the commonly applied types of methods and tools. Guidance to the applicability of these methods is provided in Section 5. In the following the 14 methods are briefly reviewed in alphabetical order:

- Data uncertainty engine (DUE)

- Error propagation equations

- Expert elicitation

- Extended peer review (review by stakeholders)

- Inverse modelling (parameter estimation)

- Inverse modelling (predictive uncertainty)

- Monte Carlo analysis

- Multiple model simulation

- NUSAP

- Quality assurance

- Scenario analysis

- Sensitivity analysis

- Stakeholder involvement

- Uncertainty matrix

References to more detailed descriptions and to supporting software tools are provided in Refsgaard et al. (2005b). For several of the methodologies more extensive descriptions are available in the RIVM/MNP Tool Catalogue, that served as a starting point for the overview presented here (Van der Sluijs et al., 2004). A summary of statistically based methods for propagation of statistical uncertainty is given by Helton and Davis (2003).

\subsection{Data uncertainty engine (DUE)}

Uncertainty in data may be described in 13 uncertainty categories (Table 2) depending on how data varies in time and space (Brown et al., 2005). Each data category is associated with a range of uncertainty models, for which more specific probability density functions (pdfs) may be developed with different simplifying assumptions (e.g. Gaussian; second-order stationarity; degree of temporal and spatial autocorrelation). Furthermore, correlation in time and space is characterised by correlogram/variogram functions. Categorical data (3) differ from numerical data $(1,2)$, because the categories are not measured on a numerical scale.

A software tool, the data uncertainty engine (DUE), for supporting the assessment of data uncertainty within the above framework has been developed within the HarmoniRiB project (Refsgaard et al., 2005c). This software tool and a report with reviews of data uncertainty for different types of data (Van Loon and Refsgaard, 2005) can be downloaded from the project website http://www.harmonirib.com.

Data uncertainty is an important input when assessing uncertainty of model outputs. Assessment of data uncertainty is an area that theoretically is complex and full of pitfalls, especially when considering the correlation structure and its link with the scale of support.

\subsection{Error propagation equations}

The error propagation equations (e.g. Mandel, 1984) are widely used in the experimental and measurement sciences

Table 2

The subdivision and coding of uncertainty-categories, along the 'axes' of space-time variability and measurement scale (Brown et al., 2005)

\begin{tabular}{lllll}
\hline Space-time variability & \multicolumn{4}{l}{ Measurement scale } \\
\cline { 2 - 5 } & $\begin{array}{l}\text { Continuous } \\
\text { numerical }\end{array}$ & $\begin{array}{l}\text { Discrete } \\
\text { numerical }\end{array}$ & Categorical & Narrative \\
\hline Constant in space and time & A1 & A2 & A3 & \\
Varies in time, not in space & B1 & B2 & B3 & 4 \\
Varies in space, not in time & C1 & C2 & C3 & \\
Varies in time and space & D1 & D2 & D3 & \\
\hline
\end{tabular}


to estimate error propagation in calculations. The error propagation equations are valid only if the following conditions are met: (1) the uncertainties have Gaussian (normal) distributions; (2) the uncertainties for non-linear models are relatively small: the standard deviation divided by the mean value is less than 0.3 ; and (3) the uncertainties have no significant covariance.

The error propagation equations for the most common operators can be seen in Box 1. The method can be extended to allow non-Gaussian distributions and to allow for co-variances.

The main advantage of the error propagation equations is that they are easy and quick to use. The key limitations lie in the underlying assumptions that seldom hold, especially not for complex calculations. The error propagation equations are therefore mainly suitable for preliminary screening analysis.

\subsection{Expert elicitation}

Expert elicitation is a structured process to elicit subjective judgements from experts. It is widely used in quantitative risk analysis to quantify uncertainties in cases where there are no or too few direct empirical data available to infer on uncertainty. Usually the subjective judgement is represented as a 'subjective' probability density function (PDF) reflecting the expert's degree of belief. Typically it is applied in situations where there is scarce or insufficient empirical material for a direct quantification of uncertainty, and where it is relevant to obtain inscrutable and defensible results (Hora, 1992).

Several elicitation protocols have been developed amongst which the much-used Stanford/SRI Protocol was the first one (Spetzler and von Holstein, 1975).

Expert elicitation typically involves the following steps: (1) Identify and select experts. (2) Explain to the expert the nature of the problem and the elicitation procedure. Create awareness of biases in subjective judgements and explore these. (3) Clearly define the quantity to be assessed and choose a scale and unit familiar to the expert. (4) Discuss the state of knowledge on the quantity at hand (strengths and weaknesses in

\section{Box 1. The error propagation equation}

The error propagation equations for the most common operators are ( $\sigma$ is the standard deviation):

Addition and Subtraction: $z=x+y+\cdots$ or $z=$ $x-y-\cdots$

$\sigma_{z}=\sqrt{\left(\sigma_{x}^{2}\right)+\left(\sigma_{y}^{2}\right)+\cdots}$

Multiplication by an exact number: $z=c x$ $\sigma_{z}=c \sigma_{x}$

Multiplication and Division: $z=x y$ or $z=x / y$

$$
\frac{\sigma_{z}}{z}=\sqrt{\left(\frac{\sigma_{x}}{x}\right)^{2}+\left(\frac{\sigma_{y}}{y}\right)^{2}+\cdots}
$$

available data, knowledge gaps, and qualitative uncertainties). (5) Elicit extremes of the distribution. (6) Assess these extremes: could the range be broader than stated? (7) Further elicit and specify the distribution (shape and percentiles or characterising parameters). (8) Verify with the expert that the distribution that you constructed from the expert's responses correctly represents the expert's beliefs. (9) Decide whether or not to aggregate the distributions elicited from different experts (this only makes sense if the experts had the same mental models of the quantity for which a distribution was elicited).

Expert elicitation has the potential to make use of all available knowledge that cannot easily be formalised otherwise. The limitations are linked to the subjectivity of the results that are sensitive to the selection of experts. In case of differences among experts it may be difficult to safely quantify the uncertainties.

\subsection{Extended peer review (review by stakeholders)}

Extended peer review is the involvement of stakeholders in the quality assurance of the modelling process. Stakeholders' reasoning, observation and imagination are not bounded by scientific rationality. This can be beneficial when tackling ill-structured, complex problems. Consequently, the knowledge and perspectives of the stakeholders can bring in valuable new views on the problem and relevant information on that problem. The latter is known as "extended facts". Stakeholders can contribute to the quality of knowledge in a number of ways. These include improvement of the quality of the problem formulation and the questions addressed by the scientists; the contribution of knowledge on local conditions which may help determine which data are strong and relevant or which response options are feasible; providing personal observations which may lead to new foci for empirical research addressing dimensions of the problem that were previously overlooked; criticism of assumptions made by the scientist, which may lead to changes towards assumptions that better match real-life conditions; and, creative thinking of mechanisms and scenarios through which projected environmental and hydrological changes may affect different sectors of society (De Marchi, 2003).

The main strength of extended peer review is that it allows the use of extra knowledge from non-scientific sources. The key limitations lie in the difficulty for stakeholders to understand the sometimes complex and abstract concepts, to ensure representativeness of the selected stakeholders and in the power asymmetries that may be reproduced.

\subsection{Inverse modelling (parameter estimation)}

Parameter values are often estimated through inverse modelling. This is also denoted as automatic calibration (Duan et al., 1994; Doherty, 2003). An optimal parameter set is sought "automatically" by minimising an objective function, often defined as the summed squared deviation between the calibration targets (field data) and their simulated counterparts. Many 
software tools support inverse modelling and some universal optimisation routines can be downloaded as freeware, e.g. PEST (Doherty, 2003) and UCODE (Poeter and Hill, 1998).

Most inversion techniques have the benefit that they in addition to optimal parameter values also produce calibration statistics in terms of parameter- and observation sensitivities, parameter correlation and parameter uncertainties. An important limitation of these parameter uncertainty techniques is that the model calibration is based on a single model (with one possible model structure). Errors in the model structure will therefore wrongly be allocated to model parameter uncertainties. The estimated parameter uncertainties are thus uncertainties for the effective model parameter given both the model structure and available observations. This also means that estimated parameter uncertainties will not compensate adequately for the model structure uncertainty, when the model is used for prediction of conditions beyond the calibration base (e.g. when calibrating on groundwater flow and subsequently using the model to simulate solute transport).

\subsection{Inverse modelling (predictive uncertainty)}

In addition to parameter estimation some of the inverse optimisation routines include the ability to estimate predictive uncertainties. The method by which the predictive uncertainty is derived varies among the inversion routines. But common to many of the local optimisation routines based on non-linear regression, is that the prediction of interest is treated as an observation, and the regression algorithm is then used to quantify the effect of the parameter uncertainty on this "observation". Some methods rely on a semi-analytical solution in which the regression algorithm is used to compute either a predictive uncertainty interval for the output variable or uncertainty in the difference between a reference case and a scenario simulation. Other methods use the regression to seek the maximum or minimum value of the prediction under the constraint that the model must be calibrated at an acceptable level, which is defined by some predefined acceptance level of the objective function.

This method provides an objective estimate of the predictive uncertainty given the applied model structure. The main limitation, apart from assumptions on linearity and normally distributed residuals, is that uncertainty can only be predicted for data types for which observations exist. This means that uncertainties on variables that are interpolated or extrapolated compared to the available field data cannot be quantified by this method.

\subsection{Monte Carlo analysis}

Monte Carlo Simulation is a statistical technique for stochastic model calculations and analysis of error propagation in calculations. Its purpose is to trace out the structure of the distributions of the model output. In its simplest form this distribution is mapped by calculating the deterministic results (realisations) for a large number of random draws from the individual distribution functions of input data and parameters of the model. As in random Monte Carlo sampling, pre-existing information about correlations between input variables can be incorporated. Monte Carlo analysis requires the analyst to specify probability distributions of all inputs and parameters, and the correlations between them. Both probability distributions and correlations are usually poorly known. Ignoring correlations and co-variance in input distributions may lead to substantial under- or over-estimation of uncertainty in model outcome. Advanced sampling methods have been designed such as Latin Hypercube sampling to reduce the required number of model runs needed to get sufficient information about the distribution in the outcome (mainly to save computation time).

A number of commercial and free software packages are available to do Monte Carlo analysis, e.g. Crystal Ball (2000) and @risk (Palisade Corporation, 2000) and SimLab (Saltelli et al., 2004). In addition Monte Carlo functionality is built into many modelling software packages. EPA (1997) provides a good guidance for use of Monte Carlo analysis.

The advantage of Monte Carlo analysis is its general applicability and that it does not impose many assumptions on probability distributions and correlations and that it can be linked to any model code. The key limitation is the large run times for computationally intensive models and the huge amount of outputs that are not always straightforward to analyse.

\subsection{Multiple model simulation}

Multiple model simulation is a strategy to address uncertainty about model structure. Instead of doing an assessment using a single model, the assessment is carried out using different models of the same system. For instance, this can be realised by having alternative model codes with different process descriptions (Linkov and Burmistrov, 2003; Butts et al., 2004) or, in the groundwater case, by having different conceptual models based on different geological interpretations (Selroos et al., 2001; Højberg and Refsgaard, 2005).

Refsgaard et al. (2006) present a new framework for dealing with uncertainty due to model structure error, based on alternative conceptual models and assessment of their pedigree and adequacy.

The main advantages of this method are that the effects of alternative model structures can be analysed explicitly and that the robustness of the model predictions increases. An important limitation is that we cannot be sure whether we have adequately sampled the relevant space of plausible models and that important plausible model structures could be overlooked.

\subsection{NUSAP}

The NUSAP system for multidimensional uncertainty assessment (Funtowicz and Ravetz, 1990; Van der Sluijs et al., 2005) aims to provide an analysis and diagnosis of uncertainty in science for policy. The basic idea is to qualify quantities by using the five qualifiers of the NUSAP acronym: numeral, unit, spread, assessment, and pedigree. NUSAP complements quantitative analysis (numeral, unit, spread) with expert judgement of reliability (assessment) and systematic multi-criteria evaluation of the different phases of production of a given 
knowledge base (pedigree). Pedigree criteria can be: proxy representation, empirical basis, methodological rigor, theoretical understanding, and degree of validation. Pedigree assessment can be further extended to also address societal dimensions of uncertainty, using criteria that address different types of value ladenness, quality of problem frames, etc. NUSAP provides insight on two independent uncertainty-related properties expressed in numbers, namely spread and strength. Spread expresses inexactness whereas strength expresses the methodological and epistemological limitations of the underlying knowledge base. The two metrics can be combined in a Diagnostic Diagram, mapping strength of for instance model parameters and sensitivity of model outcome to spread in these model parameters. Neither spread alone nor strength alone is a sufficient measure for quality. Robustness of model output to parameter strength could be good even if parameter strength is low, if the spread in that parameter has a negligible effect on model outputs. In this situation our ignorance of the true value of the parameter has no immediate consequences. Alternatively, model outputs can be robust against parameter spread even if its relative contribution to the total spread in the model is high provided that parameter strength is also high. In the latter case, the uncertainty in the model outcome adequately reflects the inherent irreducible (stochastic) uncertainty in the system represented by the model. Uncertainty then is a property of the modelled system and does not stem from imperfect knowledge on that system. Mapping components of the knowledge base in a diagnostic diagram thus reveals the weakest spots and helps in setting priorities for improvement.

The strength of NUSAP is its integration of quantitative and qualitative uncertainty. It can be used on different levels of comprehensiveness: from a 'back of the envelope' sketch based on self elicitation to a comprehensive and sophisticated procedure involving structured, informed, in-depth group discussions on a parameter by parameter format. The key limitation is that the scoring of pedigree criteria is to a large extent based on subjective judgements. Therefore, outcomes may be sensitive to the selection of experts.

\subsection{Quality assurance}

Quality assurance (QA) may be defined as protocols and guidelines to support the proper application of models. Important aims of QA are to ensure the use of best practise, to build consensus among the various actors involved in the modelling process and to ensure that the expected accuracy and model performance are in accordance with the project objectives.

Key elements of QA procedures include: (1) framing of the problem and definition of the purpose of the modelling study; (2) assessment of sources of uncertainties jointly by water manager, modeller and stakeholders and establishment of accuracy requirements by translation of the water manager and stakeholder needs to preliminary performance criteria; (3) performance of model validation tests, i.e. testing of model performance against independent data that have not been used for calibration in order to assess the accuracy and credibility of the model simulations for situations comparable to those where it is intended to be used for; and (4) reviews carried out by independent auditors with subsequent consultation between the modeller, the water manager and possibly the stakeholders at different phases of the modelling project.

Many QA guidelines exist such as Middlemis (2000) and Van Waveren et al. (1999). The HarmoniQuA project (Scholten et al., 2007; Refsgaard et al., 2005a) has developed a comprehensive set of QA guidelines for multiple modelling domains combined with a supporting software tool, MoST (downloadable via http://www.harmoniqua.org).

QA improves the chances that best practise is used, it makes it possible to involve stakeholders into the modelling process in a formalised framework, and it improves the transparency and reproducibility. If not designed and performed thoroughly, QA may become a 'rubber stamp' and generate false credibility.

\subsection{Scenario analysis}

Scenario analysis aims to describe logical and internally consistent sequences of events to explore how the future may, could or should evolve from the past and present (Van Der Heijden, 1996). The future is inherently uncertain. Different alternative futures can be explored through scenario analysis. As such, scenario analysis is also a tool to deal explicitly with different assumptions about the future.

Different types of scenarios can be distinguished. For instance, Alcamo (2001) discerns baseline vs. policy scenarios, exploratory vs. anticipatory scenarios and qualitative vs. quantitative scenarios. Baseline scenarios present the future state of society and environment in which no (additional) environmental policies do exist or have a discernible influence on society or the environment. Policy scenarios depict the future effects of environmental protection policies. Exploratory scenarios start in the present and explore possible trends into the future. Anticipatory scenarios start with a prescribed vision of the future and then work backwards in time to visualise how this future could emerge. Qualitative scenarios describe possible futures in the form of narrative texts or so-called "storylines". Quantitative scenarios provide tables and figures incorporating numerical data often generated by sophisticated models. Finally, scenarios can be surprise-free or trend scenarios, that extend foreseen developments, on the one hand or include surprises and exploring the extremes (e.g. best case/ worst case) on the other hand.

Scenarios can ensure that assumptions about future developments are made transparent and documented and are often the only way to deal with the unknown future. A limitation for qualitative scenarios is that it is difficult to test the underlying assumptions. For quantitative scenarios, the analysis is limited to those aspects of reality that can be quantified. Frequently, scenarios do not go beyond trend extrapolation and are surprise-free.

\subsection{Sensitivity analysis}

Sensitivity analysis (SA) is the study of how the variation in the output of a model (numerical or otherwise) can be 
qualitatively or quantitatively apportioned to different sources of variation, and of how the outputs of a given model depend upon the information fed into it (Saltelli et al., 2000, 2004).

Depending on the complexity of a model's output space SA methods may range from the simple to the relatively complex. If a model's output space is linear or approximates a hyperplane, SA may be conducted through a straightforward application of differential analysis. This is typically done by taking partial derivatives of the output with respect to one input, holding all other inputs constant. If a model's output space is non-linear (or does not approximate a hyperplane) then the assumptions for differential analysis do not hold. Differential analysis may be conducted, but the analyst should be aware that the results may apply only to a narrow range of the output space. For this reason, differential analysis in this situation is referred to as Local SA.

If the analyst wishes to conduct Global SA (i.e. SA across the model's entire output space) for non-linear (non-hyperplanar) models, then other analytical methods should be used. These include such methods as Monte Carlo analysis, Morris' One-at-a-time method and various variance based methods such as Fourier amplitude sensitivity test (FAST).

The strength of SA is that it provides insight in the potential influence of all sorts of changes in input and helps discrimination across parameters according to their importance for the accuracy of the outcome. A limitation is the tendency of SA to yield an overload of information. Furthermore, SA most often takes the model structure and system boundaries for granted.

\subsection{Stakeholder involvement}

Stakeholder involvement in not only the decision making process, but also in the modelling process, can help to assess and manage complex (environmental) problems in a better way. This potential can be tapped in three ways (Kloprogge and van der Sluijs, 2006): (1) by enabling them to articulate issues of concern and to improve the problem framing for research and policy; (2) by utilising their own (non-scientific) knowledge and observations and their capacity to invent new options; and (3) by involving them actively in the quality control of the operational knowledge that is co-produced (extended peer review, See section 4.4).

The RIVM/MNP guidance for uncertainty assessment and communication (Van der Sluijs et al., 2004) has a useful section on stakeholder involvement, including an instrument for discourse analysis. The HarmoniCOP project has developed a typology to characterise tools to support the public participation process in relation to the implementation of the Water Framework Directive (Maurel, 2003).

The key strengths of stakeholder involvement are that it increases the level of public accountability and it may increase the public support for implementation of subsequent management decisions.

\subsection{Uncertainty matrix}

The uncertainty matrix (Walker et al., 2003; Janssen et al., 2003) can be used to identify and prioritise the most important uncertainties in a given model study. The matrix shown in Table 3 is an example of a project specific adaptation of the more general uncertainty matrix shown in Table 1 .

For a specific application the different sources of uncertainty are listed in the rows and the type of uncertainty associated to each source is noted and characterised. This may be done either quantitatively or, as in Table 3, qualitatively. The importance of each source may then be characterised by weighting depending on its impact on the modelling study in question. The sum of uncertainty may then be assessed, e.g. by use of the error propagation equations (Section 4.2). It may not be possible to identify all sources of uncertainty and/or assigning correct weightings from the project start. The matrix may thus be reassessed at each review, where new sources of uncertainty may be added or the weight of the uncertainty adjusted as more insight into

Table 3

Example of use of the uncertainty matrix for an initial assessment of sources of uncertainty and their importance in a specific project context

\begin{tabular}{|c|c|c|c|c|c|c|}
\hline \multirow[t]{2}{*}{ Source of uncertainty } & \multicolumn{4}{|c|}{ Type of uncertainty } & \multicolumn{2}{|l|}{ Importance } \\
\hline & $\begin{array}{l}\text { Statistical } \\
\text { uncertainty }\end{array}$ & $\begin{array}{l}\text { Scenario } \\
\text { uncertainty }\end{array}$ & $\begin{array}{l}\text { Qualitative } \\
\text { uncertainty }\end{array}$ & $\begin{array}{l}\text { Recognised } \\
\text { ignorance }\end{array}$ & Weighting & (Uncertainty $\times$ weight) \\
\hline \multicolumn{7}{|l|}{ Problem context } \\
\hline - Future climate & & Medium & Medium & Large & Medium & Medium \\
\hline \multicolumn{7}{|l|}{ Input data } \\
\hline - Catchment data & Medium & & & Small & Large & Medium \\
\hline - Water quantity & Small & & & Small & Medium & Small \\
\hline - Water quality & Medium & & & Medium & Medium & Small \\
\hline \multicolumn{7}{|l|}{ Model structure (conceptual) } \\
\hline - Geology & & Large & Large & Medium & Large & Large \\
\hline - Nitrate reduction in underground & & Medium & Medium & Large & Large & Large \\
\hline \multicolumn{7}{|l|}{ Model technical uncertainty } \\
\hline
\end{tabular}


the system is gained. An uncertainty matrix used interactively during the modelling process supports the identification of all relevant sources of uncertainty and a prioritising based on a qualitative assessment of their importance. The matrix also provides a framework to keep track of all sources of uncertainty during the modelling process, so that sources identified early in the model study are not forgotten at the end of the model study, where the uncertainties are typically quantified by uncertainty simulations.

The uncertainty matrix is a good platform that may facilitate a structured dialogue between water managers, modellers and stakeholders on possible sources and types of uncertainty, which helps the key actors to approach a common understanding on the uncertainties and their importance. Its main limitation is that it strongly relies on expert judgement and mainly yields qualitative insight.

\section{Guide to select an appropriate methodology for uncertainty assessment}

Some of the more important types of methodologies and associated tools that may be applied for assessing uncertainties were briefly reviewed above. The next question is which methodology should be selected for different purposes and in different situations. This is addressed from three different perspectives in the following three subsections.

\subsection{Methodologies according to modelling process and level of ambition}

Table 4 provides a list of applicable methodologies that are considered to be adequate at different stages in the modelling process. Furthermore, it includes hints for which methodologies are more suitable for comprehensive analysis with relatively large economic resources for the study and which methodologies correspond to a lower level of ambition (denoted as "basic" in Table 4).

Uncertainty aspects are important throughout the modelling process. Considering the HarmoniQuA modelling protocol with the five steps shown in Fig. 1 and described in Section
2 above, uncertainty should be considered explicitly in all five modelling steps. However, it is treated in different ways at different stages of the modelling process. The three main actions of dealing with uncertainty may be characterised as:

- Identify and characterise sources of uncertainty. The various sources of uncertainty need to be identified and characterised in Step 1 (model study plan). This should be done by the water manager but typically after a dialogue with relevant stakeholders. Depending on the framing of the model study some of these uncertainties may be located as external non-controllable sources. It is crucial that uncertainty is considered explicitly so early in the definition phase of the model study. Here uncertainties are seldom quantified. It is also at this early stage that the first analyses are made on the acceptable level of uncertainty and the expected model performance.

- Reviews - dialogue - decisions. The last task in each of the modelling steps is a dialogue or decision task where a dialogue between water manager and modeller takes place. Often independent reviews are conducted as a basis for the decision and stakeholders and/or the general public are involved in the dialogue. As part of this dialogue, uncertainty aspects become important, e.g. when discussing whether there are sufficient data to proceed with the modelling, or whether the uncertainty of the model simulations is at a level where the results can be expected to be useful. The reviews and the stakeholder dialogues are also important platforms for a reflection on whether the assumptions made in the model are realistic and on how the study outcome may be influenced by the implicit and explicit assumptions made in the model. In many cases, more than one assumption is scientifically tenable. If such assumptions influence the model outcome, then the ignorance regarding which assumption is the best assumption can be an important source of uncertainty.

- Uncertainty assessment and propagation. Towards the end of Step 4 an uncertainty analysis should be made of the calibration and validation results. This is used for evaluating

Table 4

Suitable methodologies to deal with uncertainty at various stages of a modelling process

\begin{tabular}{llll}
\hline Type of uncertainty aspect & Step in the modelling process (cf. Fig. 1) & Level of ambition/available resources \\
\cline { 2 - 4 } $\begin{array}{l}\text { Identify and characterise } \\
\text { sources of uncertainty }\end{array}$ & Model study plan (Step 1) & Basic & Comprehensive \\
\hline Reviews-dialogue-decisions & $\begin{array}{l}\text { Review of Step 2 } \\
\text { Review of Step 3 } \\
\text { Review of Step 4 } \\
\text { Review of Step 5 }\end{array}$ & QA & EPR, QA (Update of) UM \\
& $\begin{array}{l}\text { Uncertainty analysis of calibration and } \\
\text { validation (Step 4) }\end{array}$ & DUE, EPE, SA & DUE, EPE, EE, IN-PA, IN-UN, MCA, \\
\cline { 2 - 4 } $\begin{array}{l}\text { Uncertainty assessment and } \\
\text { propagation }\end{array}$ & Uncertainty analysis of simulation (Step 5) & DUE, EPE, SA & DUE, EPE, EE, IN-UN, MCA, MMS, \\
& & & NUSAP, SC,SA, SI \\
\hline
\end{tabular}

Abbreviations of methodologies: DUE, data uncertainty; EPE, error propagation equations; EE, expert elicitation; EPR, extended peer review (review by stakeholders); IN-PA, inverse modelling (parameter estimation); IN-UN, inverse modelling (predictive uncertainty); MCA, Monte Carlo analysis; MMS, multiple model simulation; NUSAP, NUSAP; QA, quality assurance; SC, scenario analysis; SA, sensitivity analysis; SI, stakeholder involvement; UM, uncertainty matrix. 
possible biases in model simulations and assessing whether the model performance is good enough compared to the agreed accuracy requirements. Similarly, uncertainty analysis of simulations should be carried out in Step 5. Here the uncertainties in the problem framing (the context) and the management scenarios are also taken into account.

\subsection{Methodologies according to source and type of uncertainty}

Table 5 provides a list of applicable methodologies for addressing uncertainty of different types and originating from different sources. Note that the nature of uncertainty (epistemic or stochastic) has been omitted as compared to the uncertainty matrix in Table 1. The reason for this is that this is a third dimension and that each of the cells below may be divided into reducible (epistemic) and irreducible (stochastic) uncertainty.

It is noted that none of the methods covers all the cells of the table, implying that for all modelling studies a suite of uncertainty methodologies has to be selected and applied. Some more general methods, such as expert elicitation, are potentially applicable for different types and sources of uncertainty, while other more specialised methods, such as Monte Carlo analysis, are only applicable for one type (here statistical uncertainty) and a couple of sources of uncertainty.

\subsection{Methodologies according to purpose of use}

The methodologies can roughly be divided in five groups that differ in purpose of use:

- Methods for preliminary identification and characterisation of sources of uncertainty. This category is identical to the first category in Section 5.1 and the first row in Table 4. The uncertainty matrix used together with stakeholder involvement is a suitable tool for this purpose. If a first rough quantification is desired the simple error propagation equations may be suitable.
- Methods to assess the levels of uncertainty for the various sources of uncertainty. This use is addressed in some details in Section 5.2 and in Table 5. As can be seen many different methodologies may be suitable here. The exact selection will vary from case to case. It is noted from Table 5, that different methods apply to the different types of uncertainty (e.g. statistical versus qualitative uncertainty).

- Methods to propagate uncertainty through models. When all sources of uncertainty have been assessed they can be propagated through a model to assess the total uncertainty. In practise uncertainty propagation is often confined to include the data/parameters/model characteristics that have a significant effect on the total uncertainty. This selection is often supported by a sensitivity analysis. The methods suitable for uncertainty propagation are listed in the last row in Table 5. It is noted that uncertainty propagations is much easier to do for statistical and scenario uncertainty, while NUSAP and the simple error propagation equations are the only methods suitable for qualitative uncertainty (and ignorance). In practise uncertainty propagation of mixed statistical/qualitative uncertainty is very difficult to do in a rigorous manner.

- Methods to trace and rank sources of uncertainty. When the total uncertainty has been estimated it is often interesting to know how much the various sources contributed to the total uncertainty. This can be analysed by used of Monte Carlo techniques and sensitivity analysis as far as the statistical uncertainty is concerned, while NUSAP may support such analysis with respect to the more qualitative aspects.

- Methods to reduce uncertainty. When an uncertainty assessment has been made it is often desired to evaluate if some of the uncertainty can be reduced. The part of the uncertainty that is epistemic may be reduced in different ways. The classical approach in natural science is to collect more data and carry out additional studies to gain more knowledge. For modelling studies quality assurance and extended peer reviews (stakeholder involvement in the modelling process) may reduce the uncertainties as well.

Table 5

Correspondence of the methodologies with the source and types of uncertainty distinguished in the uncertainty taxonomy (inspired by Van der Sluijs et al., 2004)

\begin{tabular}{|c|c|c|c|c|c|}
\hline \multirow[t]{2}{*}{ Source of uncertainty } & & \multicolumn{4}{|c|}{ Taxonomy (types of uncertainty) } \\
\hline & & Statistical uncertainty & Scenario uncertainty & Qualitative uncertainty & Recognised ignorance \\
\hline $\begin{array}{l}\text { Context and } \\
\text { framing }\end{array}$ & $\begin{array}{l}\text { Natural, technological, } \\
\text { economic, social, political }\end{array}$ & $\mathrm{EE}$ & $\mathrm{EE}, \mathrm{SC}, \mathrm{SI}$ & $\begin{array}{l}\text { EE, EPR, NUSAP, } \\
\text { SI, UM }\end{array}$ & EE, EPR, NUSAP, SI, UM \\
\hline \multirow[t]{2}{*}{ Inputs } & System data & DUE, EPE, EE, QA & DUE, EE, SC, QA & DUE, EE & DUE, EE \\
\hline & Driving forces & DUE, EPE, EE, QA & DUE, EE, SC, QA & DUE, EE, EPR & DUE, EE, EPR \\
\hline & Parameters & IN-PA, QA & IN-PA, QA & QA & QA \\
\hline
\end{tabular}

Model output uncertainty

(via propagation)

EPE, EE, IN-UN, MCA, EE, IN-UN, MMS, SA EE, NUSAP
MMS, SA

EE, NUSAP

The bottom row lists methodologies suitable for uncertainty propagation. Abbreviations of methodologies: DUE, data uncertainty engine; EPE, error propagation equations; EE, expert elicitation; EPR, extended peer review (review by stakeholders); IN-PA, inverse modelling (parameter estimation); IN-UN, inverse modelling (predictive uncertainty); MCA, Monte Carlo analysis; MMS, multiple model simulation; NUSAP, NUSAP; QA, quality assurance; SC, scenario analysis; SA, sensitivity analysis; SI, stakeholder involvement; UM, uncertainty matrix. 


\section{Discussion and conclusions}

A terminology and typology of uncertainty is presented with the aim to assist the management of uncertainty in modelling studies for integrated water resources management. Because we focus on the use of model studies in decision making, we have adopted a subjective interpretation of uncertainty in which the degree of confidence that a decision maker has about possible outcomes and/or probabilities of these outcomes is the central focus. Other authors define the term uncertainty not as a property (state of confidence) of the decision maker but as a property (state of perfection) of the total body of knowledge or information that is available at the moment of judgement. Uncertainty is then seen as an expression of the various forms of imperfection of the available information and depends on the state-of-the-art of scientific knowledge on the problem at the moment that the decision needs to be made (assuming that the decision maker has access to the state-of-the-art knowledge). The state of perfection view goes well together with a traditional natural science basis, while our definition allows taking broader aspects of uncertainty, including those usually dealt with in social science, into account. The broader view is necessary if we want to consider all aspects of modelling uncertainty when modelling is used as an element in the broader water management process.

We have briefly reviewed 14 methods for assessing and characterising uncertainty. These methods are very different in nature, some originating from the statistical world, while others have their roots in social science. The 14 methods have been mapped against a framework for the modelling process, its interaction with the broader water management process and the role of uncertainty at different stages in the modelling processes.

Numerous methods that deal with uncertainty exist. The 14 methods we have included are by no means exhaustive, but intend to present a representative cross-section of commonly applied methods covering the various aspects of uncertainty in water resources management. Many methods reported in literature naturally fall within one of the 14 'boxes', while others fall in between. An example of a method that does not fit well to our selection of methods is the generalised uncertainty likelihood estimation (GLUE) method (Beven and Binley, 1992; Beven, 2002). GLUE can be used both as a kind of calibration method or as an uncertainty propagation method. It is based on the concept of equifinality and can be seen as a method having similarities in approach with three of the above 14 methods: Inverse modelling (parameter estimation), Monte Carlo analysis and multiple model simulation. Similarly many software tools have functionality corresponding to a couple of the 14 methods.

None of these methodologies is applicable to address all the different relevant aspects of uncertainty in the modelling in relation to water resources management. Most of the methods we have selected are complementary in approaches and content. However, there are also some important overlaps. The best example of that is the quality assurance method that in reality is a framework within which some of the other methods, such as stakeholder involvement and extended peer review are typically recommended. In the quality assurance tool MoST
(Refsgaard et al., 2005a; Scholten et al., 2007) all other methods are incorporated.

The key conclusion of the analysis in this paper is that uncertainty assessment is not just something to be added after the completion of the modelling work. Instead uncertainty should be seen as a red thread throughout the modelling study starting from the very beginning. Traditionally, uncertainty assessments are carried out only at the end of a modelling study when the models have been calibrated and validated. Standard techniques, often included in the model GUIs, are then used to propagate and quantify the uncertainty, e.g. sensitivity analysis or Monte Carlo analysis. The major argument towards this type of uncertainty assessments is that the standard techniques do typically only address one type of uncertainty, namely the statistical uncertainty. By performing the uncertainty analysis as an 'add-on' by standard techniques in the end of the model study, and report this as the uncertainty analysis, it is implicitly assumed that the statistical uncertainty is the most important uncertainty. The statistical uncertainty does, however, only comprise a limited space of the total uncertainty, as illustrated in Fig. 3. Moving towards the use of models in a broader perspective, such as water management plans and the participatory processes in the WFD, other types of uncertainty emerged that have not traditionally been addressed in a model study. It is therefore crucial that the uncertainty assessment is introduced in the introductory phase and tracked throughout the model study and that the identification and characterisation of all uncertainty sources are performed jointly by the modeller, the water manager and stakeholders in connection with the problem framing and identification of the objectives of the modelling study.

\section{Acknowledgement}

The present work was carried out within the Concerted Action Harmoni-CA, which is partly funded under EC's 5th Framework Research Programme (Contract EVK1-CT2001-00192). A previous, and much longer, version of the work can be found in a report (Refsgaard et al., 2005b) which emerged after formal external reviews and discussions at a CATCHMOD Technical Workshop, Copenhagen 16.11.2004. The constructive comments of two anonymous reviewers are acknowledged.

\section{References}

Alcamo, J., 2001. Scenarios as Tools for International Environmental Assessments. European Environment Agency, Copenhagen. Environmental Issues Report. Experts Corner Report. Prospects and Scenarios No. 5.

Beck, M.B., 1987. Water quality modelling: a review of the analysis of uncertainty. Water Resources Research 23 (8), 1393-1442.

Beven, K., Binley, A.M., 1992. The future of distributed models, model calibration and uncertainty predictions. Hydrological Processes 6, 279-298.

Beven, K., 2002. Towards a coherent philosophy for modelling the environment. Proceedings of the Royal Society of London, A 458 (2026), 2465-2484.

Brown, J.D., 2004. Knowledge, uncertainty and physical geography: towards the development of methodologies for questioning belief. Transactions of the Institute of British Geographers 29 (3), 367-381.

Brown, J.D., Heuvelink, G.B.M., Refsgaard, J.C., 2005. An integrated framework for assessing and recording uncertainties about environmental data. Water Science and Technology 52 (6), 153-160. 
Butts, M.B., Payne, J.T., Kristensen, M., Madsen, M., 2004. An evaluation of the impact of model structure on hydrological modelling uncertainty for streamflow simulation. Journal of Hydrology 298, 242-266.

Crystal Ball, 2000. User Manual. Decision Engineering Inc, Denver.

De Marchi, B., 2003. Public participation and risk governance. Science and Public Policy 30 (3), 171-176.

Doherty, J., 2003. Ground water model calibration using pilot points and regularization. Ground Water 41 (2), 170-177 (See also http://www.sspa. com/pest for further information/download of PEST).

Duan, Q., Sorooshian, S., Gupta, V.K., 1994. Optimal use of the SCE-UA global optimization method for calibrating watershed models. Journal of Hydrology 158, 265-284.

EC, 2000. Directive 2000/60/EC of the European Parliament and of the Council of October 232000 Establishing a Framework for Community Action in the Field of Water Policy. Official Journal of the European Communities, L327/1-L327/72. 22.12.2000.

EC, 2003. Common Implementation Strategy for the Water Framework Directive (2000/60/EC). Guidance Document No. 11. Planning Processes. Working Group 2.9. Office for the Official Publications of the European Communities, Luxembourg.

EPA, 1997. Risk Assessment Forum, Guiding Principles for Monte Carlo Analysis, EPA/630/R-97/001. (http://www.epa.gov/NCEA/pdfs/montcarl.pdf).

Funtowicz, S.O., Ravetz, J., 1990. Uncertainty and Quality in Science for Policy. Kluwer Academic Publishers, Dordrecht.

GWP, 2000. Integrated Water Resources Management. TAC Background Papers No. 4. Global Water Partnership, Stockholm.

Helton, J.C., Davis, F.J., 2003. Latin Hypercube sampling and the propagation of uncertainty analysis of complex systems. Reality Engineering and System Safety (81), 23-69.

Henriksen, H.J., Refsgaard, J.C., Højberg, A.L., Ferrand, N., Gijsbers, P., Scholten, H., Public participation in relation to quality assurance of water resources modelling (HarmoniQuA). (submitted for publication).

Hora, S.C., 1992. Acquisition of expert judgement: examples from risk assessment. Journal of Energy Engineering 118, 136-148.

Højberg, A.L., Refsgaard, J.C., 2005. Model Uncertainty - parameter uncertainty versus conceptual models. Water Science and Technology 52 (6), 177-186.

Jakeman, A.J., Letcher, R.A., 2003. Integrated assessment and modelling: features, principles and examples for catchment management. Environmental Modelling \& Software 18, 491-501.

Janssen, P.H.M., Petersen, A.C., van der Sluijs, J.P., Risbey, J.S., Ravetz, J.R., 2003. RIVM/MNP Guidance for Uncertainty Assessment and Communication: Quickscan Hints \& Actions List. RIVM/MNP, ISBN 90-6960-105-2. Available from: www.nusap.net.

Jønch-Clausen, T., 2004. Integrated Water Resources Management (IWRM) and Water Efficiency Plans by 2005. Why, What and How? Global Water Partnership, TEC Background Papers No. 10, Stockholm.

Klauer, B., Brown, J.D., 2004. Conceptualising imperfect knowledge in public decision making: ignorance, uncertainty, error and 'risk situations'. Environmental Research, Engineering and Management 27 (1), 124-128.

Linkov, I., Burmistrov, D., 2003. Model uncertainty and choices made by the modelers: lessons learned from the international atomic energy agency model intercomparisons. Risk Analysis 23 (6), 1297-1308.

Kloprogge, P., van der Sluijs, J., 2006. The inclusion of stakeholder knowledge and perspectives in integrated assessment of climate change. Climatic Change 75 (3), 259-389.

Mandel, J., 1984. The Statistical Analysis of Experimental Data. Dover Publications, New York, USA, 410 pp.

Maurel, P., (Ed.), 2003. Public participation and the European Water Framework Directive. Role of Information and Communication Tools. Cemagref, Montpellier, Workpackage 3 Report of the HarmoniCOP Project. www.harmonicop.info.

Middlemis, H., 2000. Murray-Darling Basin Commission. Groundwater Flow Modelling Guideline. Aquaterra Consulting Pty Ltd, South Perth, Western Australia. Project no. 125.

Pahl-Wostl, C., 2002. Towards sustainability in the water sector - the importance of human actors and processes of social learning. Aquatic Sciences 64, 394-411.

Pahl-Wostl, C., 2007. The implications of complexity for integrated resources management. Environmental Modelling \& Software 22, 561-569.
Palisade Corporation, 2000. Guide to Using @ RISK - Risk Analysis and Simulation Add-in for Microsoft Excel, Version 4, March 2000.

Pascual, P., Steiber, N., Sunderland, E., 2003. Draft Guidance on Development, Evaluation and Application of Regulatory Environmental Models. The Council for Regulatory Environmental Modeling. Office of Science Policy, Office of Research and Development. US Environmental Protection Agency, Washington DC, 60 pp.

Poeter, E.P., Hill, M.C., 1998. Documentation of UCODE, A Computer Code for Universal Inverse Modelling. U.S. Geological Survey, Water-Resources Investigations Report 98-4080. (Available from www.usgs.gov/software/ code/ground_water/ucode).

Refsgaard, J.C., Henriksen, H.J., 2004. Modelling guidelines - terminology and guiding principles. Advances in Water Resources 27, 71-82.

Refsgaard, J.C., Henriksen, H.J., Harrar, W.G., Scholten, H., Kassahun, A., 2005a. Quality assurance in model based water management - review of existing practice and outline of new approaches. Environmental Modelling \& Software 20, 1201-1215.

Refsgaard, J.C., van der Sluijs, J.P., Højberg, A.L., Vanrolleghem, P.A., 2005 b. Uncertainty Analysis. Harmoni-CA Guidance No. 1, 46 pp. Downloadable from www.harmoni-ca.info.

Refsgaard, J.C., Nilsson, B., Brown, J., Klauer, B., Moore, R., Bech, T., Vurro, M., Blind, M., Castilla, G., Tsanis, I., Biza, P., 2005c. Harmonised techniques and representative river basin data for assessment and use of uncertainty information in integrated water management (HarmoniRiB). Environmental Science and Policy 8, 267-277.

Refsgaard, J.C., van der Sluijs, J.P., Brown, J., van der Keur, P., 2006. A framework for dealing with uncertainty due to model structure error. Advances in Water Resources 29, 1586-1597.

Saltelli, A., Chan, K., Scott, M., 2000. Sensitivity Analysis. John Wiley \& Sons publishers. Probability and Statistics Series.

Saltelli, A., Tarantola, S., Campolongo, F., Ratto, M., 2004. Sensitivity Analysis in Practice: A Guide to Assessing Scientific Models. John Wiley \& Sons Publishers.

Scholten, H., Kassahun, A., Refsgaard, J.C., Kargas, T., Gavardinas, C., Beulens, A.J.M., 2007. A methodology to support multidisciplinary modelbased water management. Environmental Modelling \& Software 22, 743-759.

Selroos, J.O., Walker, D.D., Strom, A., Gylling, B., Follin, S., 2001. Comparison of alternative modelling approaches for groundwater flow in fractured rock. Journal of Hydrology 257, 174-188.

Spetzler, C.S., von Holstein, S., 1975. Probability encoding in decision analysis. Management Science 22 (3), 340-358.

Vandenberghe, V., Bauwens, W., Vanrolleghem, P.A., 2007. Evaluation of uncertainty propagation into river water quality predictions to guide future monitoring campaigns. Environmental Modelling \& Software 22, 725-732.

Van Der Heijden, K., 1996. Scenarios: The Art of Strategic Conversation. John Wiley \& Sons, ISBN 0471966398.

Van der Sluijs, J.P., Janssen, P.H.M., Petersen, A.C., Kloprogge, P., Risbey, J.S., Tuinstra, W., Ravetz, J.R., 2004. RIVM/MNP Guidance for Uncertainty Assessment and Communication Tool Catalogue for Uncertainty Assessment. Utrecht University (Downloadable from. http:// www.nusap.net/sections.php?op=viewarticle $\& ; \operatorname{artid}=17)$.

Van der Sluijs, J.P., Craye, M., Funtowicz, S., Kloprogge, P., Ravetz, J., Risbey, J., 2005. Combining quantitative and qualitative measures of uncertainty in model based environmental assessment: the NUSAP System. Risk Analysis 25 (2), 481-492.

Van Loon, E., Refsgaard, J.C. (Eds.), 2005. Guidelines for Assessing Data Uncertainty in River Basin Management Studies. Geological Survey of Denmark and Greenland, Copenhagen, pp. 182. Available on http:// www.harmonirib.com.

Van Waveren, R.H., Groot, S., Scholten, H., Van Geer, F., Wösten, H., Koeze, R., Noort, J., 1999. Good Modelling Practice Handbook, STOWA. Utrecht, The Netherlands (in Dutch). English version from http://www.landandwater.tudelft.nl/Downloads/GMP-uk.pdf.

Walker, W.E., Harremoës, P., Rotmans, J., Van der Sluijs, J.P., Van Asselt, M.B.A., Janssen, P., Krayer von Krauss, M.P., 2003. Defining uncertainty a conceptual basis for uncertainty management in model-based decision support. Integrated Assessment 4 (1), 5-17.

Weiss, C., 2003. Expressing scientific uncertainty. Law, Probability and Risk (2), 25-46. 